\title{
BATERIA MULTIDISCIPLINAR DE TRIAGEM DO DESEN- VOLVIMENTO INFANTIL: ESTUDOS PSICOMÉTRICOS DO INSTRUMENTO DE AVALIAÇÃO DO DESENVOLVIMENTO DAS HABILIDADES MOTORAS
}

\section{APPLICATION OF MOTOR DEVELOPMENT SCALE IN CHILDREN WITH AUTISM SPECTRUM DISORDERS: AN EXPLORATORY STUDY}

Flavia Amaral Machado (ORCID: 0000-0001-7398- 8759) $)^{1,2}$ Sérgio Kakuta Kato (ORCID: 0000-0002-7180-3121) Emanuelle Plotsky de Castro (ORCID: 0000-0002-4891-1829) ${ }^{1}$ Léia Gonçalves Gurgel (ORCID: 0000-0003-2679-1798) Adriana Jung Serafini (ORCID: 0000-0002-9273-5594) 1,2,4 Caroline Tozzi Reppold (ORCID: 0000-0002-0236-2553) 1,2,3,4

Autor Correspondente Flavia Amaral Machado E-mail: flaviaamaralmachado@gmail. com

${ }^{1}$ Laboratório de Pesquisa em Avaliação Psicológica. Universidade Federal de Ciências da Saúde de Porto Alegre.

2 Programa de Pós-Graduação em Ciências da Saúde. Universidade Federal de Ciências da Saúde de Porto Alegre.

${ }^{3}$ Programa de Pós-Graduação em Ciências de Reabilitação. Universidade Federal de Ciências da Saúde de Porto Alegre.

${ }^{4}$ Programa de Pós-Graduação em Psicologia e Saúde. Universidade Federa de Ciências da Saúde de Porto Alegre.

a) Financiamento:

O presente trabalho foi realizado com apoio do CNPq e FAPERGS

b) Este estudo é derivado da Tese de Doutorado de Flavia Amaral Machado intitulada "Elaboração, validação e normatização de uma bateria multidisciplinar infantil", apresentada ao Programa de Pós-graduação em Ciências da Saúde da Universidade Federal de Ciências da Saúde de Porto Alegre, concluído em agosto de 2019.

\section{RESUMO}

Objetivo: apresentar a estimativa de precisão e as evidências de validade baseada nas relações com variáveis externas do Teste de Triagem do Desenvolvimento Infantil - Habilidades Motoras (TDI-HM). Esse instrumento compõe uma bateria multidisciplinar de triagem que avalia, além das Habilidades Motoras, a Linguagem e as Funções Executivas de crianças em fase de escolarização inicial. Métodos: a amostra do estudo foi composta por 382 alunos (6 a 8 anos), matriculados em escolas públicas de Porto Alegre, Rio Grande do Sul, Brasil. As variáveis do desenvolvimento motor foram rastreadas pelo instrumento elaborado (TDI-HM) e pelo teste Escala de Desenvolvimento Motor (EDM). Resultados: a análise de fidedignidade do TDI-HM indicou boa consistência interna do total de 16 itens do instrumento $($ Alfa $=0,76)$. A consistência interna (Alfa) das dimensões avaliadas em separado apresentou: motricidade ampla $=0,84$ ( 6 itens $)$, equilíbrio $=0,67$ (4 itens), motricidade fina $=0,70$ ( 6 itens). Comparando desempenho na escala por idade, os resultados indicaram que houve diferença significativa das médias por idade tanto no quociente motor geral - 6 anos, 110,50 (DP 7,86); 7 anos 100,21(DP 5,57) e 8 anos 89,85 (4,28) - quanto por dimensão. Conclusões: os resultados apresentaram evidências de que o TDI-HM tem potencial para verificar se o desempenho das HM está de acordo com os parâmetros esperados para a idade. Considera-se que, na sequência dos estudos, isso possa contribuir para o uso em pesquisas, em práticas clínicas e escolares relacionadas com o desenvolvimento motor em crianças no Brasil.

Palavras-chave: Habilidade Motora; Estudos de Validação; Desenvolvimento Infantil.

\begin{abstract}
Objective: this work aimed at searching for estimate of precision and validity evidences based on the relation with external variables to Child Development Screening Test - Motor Skills (TDI-HM). This is a tool in a multidisciplinary screening battery that assesses Motor Skills, Language, and Executive Functions of children in the schooling early phase. Methods: the sample was 382 students (6 to 8 years old), from Porto Alegre public schools. The instruments used for the motor skills development were TDI-HM and the Motor Development Scale (EDM) Results: the TDI-HM reliability analysis indicated acceptable coefficients of the all16 items of the instrument $($ Alpha $=0.76)$. The internal consistency (Alpha) of the dimensions assessed separately was: Global Motricity $=0.84(6$ items), Balance $=0.67$ (4 items), Fine Motricity $=0.70$ (6 items). Comparing performance on the age scale, the results indicated that there was a significant difference in the means for age in both the general motor quotient (QM): 6 years 110.50 (SD 7.86); 7 years 100.21 (SD 5.57); and 8 years 89.85 (4.28), as specific quotient. Conclusion: the results showed evidence that the TDI-HM has the potential to verify if the performance of the HMs is in accordance with the expected parameters for age. The TDI-HM can contribute and be used in research, clinical, and school practices related to motor development in Brazilian children.
\end{abstract}

Keywords: Motor Skill; Validation Studies; Child Development. 


\section{INTRODUÇ̃̃̃O}

O período compreendido entre o nascimento e o final do primeiro ano de vida da criança é considerado uma das etapas mais críticas no desenvolvimento infantil, pois, nela, o Desenvolvimento Motor (DM) apresenta um ritmo acelerado de mudanças que culminam nas funções de mobilidade, como a aquisição do sentar, do engatinhar e da marcha independente ${ }^{1,2}$. Algumas Habilidades Motoras (HM), como os ajustes da postura corporal, permanecem em desenvolvimento até a vida adulta $^{2}$

Os movimentos podem ser entendidos em diversos níveis. Na base do DM, encontram-se os movimentos reflexos ou involuntários, envolvidos nas questões de sobrevivência; em seguida, os movimentos rudimentares, apresentando-se como as primeiras formas de movimentos voluntários. Após esse período, ocorre o desenvolvimento das HM relacionadas com as ações locomotoras, manipulativas e estabilizadoras ${ }^{3,4}$ - que é a maior entre as fases do DM da infância, compreendendo o período de 2 a 6 anos de idade aproximadamente, na qual o indivíduo passa por três estágios distintos: inicial, elementar e maduro.

A partir desses estágios mais básicos, desenvolvem-se as HM nas quais os movimentos apresentam maior nível de controle e precisão. Com elas, o indivíduo é capaz de desenvolver habilidades especializadas e de realizar atividades específicas, de maneira mais aprimorada ${ }^{3,4}$. As HM subdividem-se, de acordo com seu grau de complexidade, em Amplas - como o controle postural e o deslocamento - e Finas - como a manipulação de objetos e a escrita ${ }^{1-3}$. A possibilidade de executar movimentos que permitam à criança controlar e explorar o ambiente ao seu redor propiciará, no futuro, a capacidade de utilizar adequadamente o papel, o lápis e os demais recursos educacionais utilizados na escolarização ${ }^{5}$.
A etapa de início da complexificação das HM coincide com o período de escolarização inicial ${ }^{6}$. Desse modo, os componentes da aprendizagem motora exercem influência significativa na aquisição das habilidades de aprendizagem cognitiva, como a consciência corporal, direcional e espacial, bem como sincronia, ritmo e sequência motora ${ }^{6}$. Diversos estudos apresentam a associação entre dificuldades motoras e déficits de aprendizagem $^{1,6,7}$ e evidenciam que déficits motores podem comprometer atividades cotidianas da criança ${ }^{8}$, prejudicar suas interações sociais e, consequentemente, comprometer sua autoestima e seu autoconceito ${ }^{9,10}$.

Devido à importância das HM para o desenvolvimento físico, cognitivo, social e emocional do indivíduo ${ }^{6}$, surge a necessidade de estabelecer métodos adequados capazes de rastrear possíveis atrasos ou desordens do desenvolvimento. A utilização de um instrumento que permita avaliar um maior número de crianças, de fácil aplicação e que seja livre de custo é uma possibilidade viável para proporcionar intervenções precoces e evitar ou amenizar possíveis déficits em outras esferas da vida da criança ${ }^{5,6,13}$.

Embora ocorra um incremento dos estudos nessa área nos últimos anos, ainda há carência de instrumentos avaliativos com boas propriedades psicométricas e adaptados para a população brasileira13. Visando preencher essa lacuna, foi elaborado o instrumento de avaliação de DM, o Teste de Triagem do Desenvolvimento Infantil - Habilidades Motoras (TDI-HM), que integra uma bateria multidisciplinar para avaliação do desenvolvimento de crianças em fase de escolarização inicial. Especificamente para este estudo, o objetivo principal foi apresentar novas características psicométricas do TDI-HM, analisando a estimativa de precisão e as evidências de validade baseadas em variáveis externas. 


\section{MÉTODOS}

\section{Definição do construto e das dimensões}

Especificamente, este estudo refere-se ao instrumento para avaliação do DM, considerado no estudo como um processo sequencial e contínuo relativo à idade cronológica, que compreende motricidade ampla, equilíbrio e motricidade fina. É parte de pesquisa intitulada "Elaboração, Validação e Normatização de um Instrumento de Avaliação Multidisciplinar Infantil, composto por 3 domínios: Linguagem, Funções Executivas (FEs) e Habilidades Motoras (HMs)".

Para ESTE estudo, definiu-se como HM Ampla ou motricidade ampla a capacidade de realizar movimentos amplos e coordenados, os quais envolvem grandes grupos musculares ${ }^{3}$. O equilíbrio foi definido como a capacidade do corpo em assumir e sustentar sua posição contra a força da gravidade, sendo a habilidade de manutenção de uma postura do corpo estático ou durante a execução de um movimento (equilíbrio dinâmico) $^{3}$. Por sua vez, a HM Fina ou motricidade fina foi definida para o estudo como a capacidade de controlar um conjunto de atividades de movimento de alguns segmentos do corpo, com emprego de força mínima, para realizar uma tarefa com precisão. São habilidades de pequenos músculos do corpo, como os músculos dos dedos, do punho; e pela destreza manual (como escrever, desenhar, costurar etc.) ${ }^{3}$.

\section{Amostra do estudo}

A amostra foi composta por 382 alunos de escolas públicas do município de Porto Alegre, Rio Grande do Sul, Brasil. Os estudantes estavam regularmente matriculados do $1^{\circ}$ ao $3^{\circ}$ ano do Ensino fundamental I (EFI), caracterizado, a partir da literatura, como fase de escolarização inicial ${ }^{14,15}$. Os critérios de seleção foram: estar regularmente matriculado em escola pública de Porto Alegre, ter idade de 6 a 8 anos e 11 meses e apresentar um Termo de Consentimento Livre e Esclarecido assinado por um de seus cuidadores/ responsáveis. Os critérios de exclusão foram: apresentar algum tipo de comprometimento neurológico ou físico (de acordo com o prontuário fornecido pela escola); por algum motivo, não concluir a bateria de testes. A investigação respeitou todos os preceitos éticos próprios de pesquisa com seres humanos em acordo com as Resoluções no 466/2012 e n n $^{\text {5 }}$ 10/2016 do Conselho Nacional de Saúde e obteve aprovação do Comitê de Ética em Pesquisa da Universidade Federal de Ciências da Saúde de Porto Alegre, sob o parecer $n^{\circ}$ 2.619.678.

\section{Instrumentos}

TDI-HM - Este instrumento compõe uma Bateria de triagem multidisciplinar. É composto por 5 tarefas (16 itens), cada uma delas com crescente grau de complexidade. Envolve a avaliação das seguintes dimensões do DM: motricidade ampla, equilíbrio e motricidade fina. De acordo com os resultados obtidos em cada dimensão, é calculado um Quociente Motor (QM), considerando o resultado das tarefas (pontuação total) dividido pela idade cronológica e multiplicado por 100. De acordo com esse QM, é possível classificar o desempenho motor do indivíduo como "acima do esperado para a idade", "esperado para a idade" ou "abaixo do esperado para a idade".

A avaliação da motricidade ampla é realizada por meio de duas tarefas, cada uma é composta por três itens, ambas com uso de bola (circunferência de 65 a $68 \mathrm{~cm}$ ). A primeira refere-se ao movimento de quicar a bola por três vezes. Na segunda, o participante simula o movimento de arremesso em cesta de basquete. $\mathrm{O}$ avaliador observa a postura da criança durante a execução dos movimentos, considerando os três segmentos: membros superiores, tronco e membros inferiores. Para cada um, há três alternativas de resposta de acordo com a postura apresentada durante o movimento. $\mathrm{O}$ avaliador marca com um $\mathrm{X}$ ao lado da figura correspondente ao estágio do desenvolvimento apresentado pelo participante. Para cada estágio, é atribuída uma faixa etária: inicial (6 anos), elementar (7 anos) ou maduro (8 anos). Nessa atividade, há três tentativas, e considera-se a maior 
exatidão ao exercício, ou seja, a melhor das três tentativas. As duas tarefas são previamente demonstradas pelo avaliador.

O equilíbrio é avaliado em uma tarefa composta por uma sequência de saltos, proposta de acordo com a idade da criança: salto com apoio nos membros inferiores bipodal e unipodal (6 anos); salto com apoio unipodal sobre o mesmo membro inferior ( 7 anos); e giro sem apoio de $180^{\circ}$ ( 8 anos). A execução é realizada em um tapete com medidas específicas, de layout semelhante ao jogo "amarelinha". Em cada item, o avaliador marca no protocolo se o indivíduo conseguiu realizar o movimento ou não. A pontuação final indica qual o nível de desempenho (dificuldade) alcançado. Durante a execução da tarefa, a criança dispõe de três tentativas, sendo verificada a exatidão do movimento por parte do avaliador. A tarefa é demonstrada pelo avaliador previamente.

A avaliação da motricidade fina compreende duas tarefas. A primeira envolve um traçado gráfico (3 itens), composto por linha reta ( 6 anos), linha curva (7 anos) e espiral (8 anos). Para realização da tarefa, o participante deve executar o traçado na linha pontilhada com a maior exatidão possível. A segunda tarefa, envolve recorte de papel (3 itens) e é diferenciada conforme a idade da criança. Compreende o recorte (seguindo a linha preta do desenho) de linha reta (6 anos), linha curva (7 anos) e linha ondulada (8 anos). Em cada tarefa, são três níveis de dificuldades diferentes, atribuídos a diferentes faixas-etária, sendo que, nesses exercícios, são considerados os números de inconformidades produzidas em relação às instruções fornecidas.

Escala do Desenvolvimento Motor $(E D M)^{16}-\mathrm{O}$ instrumento avalia aspectos do DM, como motricidade fina, equilíbrio, esquema corporal, organização espacial, organização temporal e a lateralidade ${ }^{17}$. Para este estudo, utilizou-se a bateria de testes relacionados com as variáveis motricidade global, motricidade fina e equilíbrio, sendo composta por 30 itens (10 itens para cada dimensão). Calcula-se o Quociente Motor Geral (QMG) da criança, relacionado com a classificação das habilidades, de acordo com os índices padrões a seguir: muito superior (130 ou mais); superior (120-129); normal alto (110-119); normal médio (90-109); normal baixo (80$89)$; inferior (70-79); e muito inferior (69 ou menos) ${ }^{17}$.
No presente estudo, a EDM foi utilizada com o objetivo de estabelecer evidências de validade da TDI-HM baseada nas relações com variáveis externas convergentes. $\mathrm{O}$ instrumento foi escolhido como correlato por ser um dos poucos instrumentos elaborados para a população brasileira, que contemplem a faixa etária deste estudo, e por possuir os mesmos construtos avaliados na TDI-HM.

\section{Procedimentos}

A coleta de dados foi realizada em dois encontros individuais, em dias diferentes. As atividades ocorreram no horário regular de aula, de acordo com a disponibilidade de cada turma, e foram conduzidas por avaliadores previamente treinados na aplicação dos instrumentos por um fisioterapeuta da equipe de pesquisa. As respostas foram anotadas pelos entrevistadores nos protocolos de aplicação próprios de cada instrumento, e cada protocolo foi corrigido e revisado por dois avaliadores independentes, rigorosamente treinados na aplicação e correção dos dados dos instrumentos. Todos os protocolos tiveram a correção e a digitação revisadas por um terceiro avaliador.

\section{Análise dos dados}

A análise de dados foi feita com base na Teoria Clássica dos Testes $(\mathrm{TCT})^{18,19}$. Para verificar a fidedignidade do instrumento, foi avaliada a consistência interna dos dados coletados a partir da obtenção do coeficiente Alfa de Cronbach. A busca de evidências de validade baseada na relação com variáveis externas, comparando desempenho na escala por idade (6, 7 e 8 anos), foi feita por meio de Análise de Variância (ANOVA). A suposição de normalidade dos dados foi verificada por meio do teste Kolmogorov Smirnov. A evidência de validade convergente foi verificada com a utilização do Coeficiente de Correlação de Pearson entre os escores por construto do instrumento elaborado (TDI-HM) e a $\mathrm{EDM}^{16}$. Foi adotado nível de significância $5 \%$ para todas as comparações. Para a análise estatística dos dados coletados, foi utilizado o programa SPSS - Statistical Package for Social Science, versão 23.0. 


\section{RESULTADOS}

Os dados referentes à caracterização da amostra estão apresentados na tabela 1 .

Tabela 1. Distribuição das variáveis de caracterização da amostra. $(\mathrm{n}=382)$

\begin{tabular}{|c|c|c|}
\hline & Frequência & Porcentagem \\
\hline \multicolumn{3}{|l|}{ Sexo } \\
\hline Feminino & 229 & 59,9 \\
\hline Masculino & 153 & 40,1 \\
\hline \multicolumn{3}{|l|}{ Idade } \\
\hline 6 anos & 114 & 29,8 \\
\hline 7 anos & 134 & 35,1 \\
\hline 8 anos & 134 & 35,1 \\
\hline \multicolumn{3}{|l|}{ Escolaridade } \\
\hline $1^{\circ}$ ano & 169 & 44,3 \\
\hline $2^{\circ}$ ano & 134 & 35,0 \\
\hline $3^{\circ}$ ano & 79 & 20,7 \\
\hline
\end{tabular}

Para os resultados de motricidade ampla, foi considerado o QM de acordo com a idade e com o estágio do desenvolvimento nos três segmentos (membros superiores, tronco e membros inferiores). As respostas possíveis eram A (estágio inicial), B (estágio elementar) e C (estágio maduro); e foram atribuídas, respectivamente, às idades 6,7 e 8 anos. A média das idades atribuídas às questões foi dividida pela idade cronológica e multiplicada por 100.

A tarefa de equilíbrio foi composta por quatro itens e, da mesma forma que a motricidade ampla, apresentou um índice em relação à idade e ao nível de dificuldade executado. Foi atribuída idade a cada nível de execução correta das atividades, sendo 6 anos para as crianças que conseguiram executar as atividades M1 e M2, 7 anos para execuções até M3 e 8 anos para as crianças que executaram todas as atividades. Para o cálculo do quociente de equilíbrio, a idade obtida nas tarefas foi dividida pela idade cronológica e multiplicada por 100 .
Nas tarefas de motricidade fina, considerou-se como resultado o número de afastamentos da linha do traçado e da linha de recorte, dos três níveis de dificuldade, sendo que, quanto maior o número de afastamentos, pior o desempenho. Tendo como base estudos em que a prevalência de crianças da faixa etária de 5 a 10 anos com dificuldade de movimento ou risco de dificuldade é menor que $25 \% 20$ -22 , criou-se uma tabela com os escores esperados por idade considerando os $25 \%$ com pior desempenho para cada idade. A normatização da pontuação foi realizada para que os resultados da escala pudessem ser comparados entre crianças de diferentes idades. Para a obtenção da normatização da pontuação, considerou-se o percentil 75 do escore bruto (soma do número de afastamentos de cada item de M11 a M16). A média das idades atribuídas às questões foi dividida pela idade cronológica e multiplicada por 100, obtendo assim o quociente de motricidade fina. A normatização é apresentada na tabela 2 . 
Tabela 2. Motricidade Fina: distribuição por idade do número de afastamentos, considerando percentil 75

\begin{tabular}{cccc}
\hline \multirow{2}{*}{ Motricidade Fina } & \multicolumn{3}{c}{ Idade (anos) } \\
\cline { 2 - 4 } & $\mathbf{6}$ & $\mathbf{7}$ & $\mathbf{8}$ \\
\hline Itens Traçado & 6 & 5 & 4 \\
\hline M11 & 8 & 7 & 6 \\
M12 & 10 & 9 & 8 \\
\hline M13 & & & \\
\hline Itens Recorte & 2 & 1 & 0 \\
\hline M14 & 6 & 4 & 2 \\
M15 & 3 & 2 & 1 \\
M16 & & & \\
\hline
\end{tabular}

O resultado da análise de fidedignidade do TDI-HM total inclui os 16 itens e indicou boa consistência interna. $\mathrm{O}$ índice calculado por meio do coeficiente Alfa de Cronbach foi de $0,76(\mathrm{p}=0,001)$. Os valores de alfa $(p=0,001)$ obtidos nas dimensões avaliadas foram: motricidade ampla $=0,84$ (muito boa), equilíbrio $=0,67$ (aceitável) e motricidade fina $=0,70$ (boa).

Em relação aos resultados do QM, houve diferença significativa das médias por idade tanto no QM geral quanto por dimensão (tabela 3). Para traçar um perfil motor da população avaliada, os escores brutos do QM foram classificados em "abaixo do esperado para a idade", "esperado para a idade" e "acima do esperado para a idade". Utilizando os mesmos parâmetros de Rosa-Neto na EDM16, considerou-se que valores abaixo de 89 despertam atenção dos profissionais de saúde, com risco leve para atraso do movimento. Na tabela 4, é apresentada a classificação do QM por idade. 
Tabela 3. Quociente Motor Geral e por construto

\begin{tabular}{|c|c|c|c|c|c|c|c|c|}
\hline \multirow{3}{*}{ Quociente } & \multicolumn{6}{|c|}{ Idade (anos) } & \multicolumn{2}{|c|}{ Total } \\
\hline & \multicolumn{2}{|c|}{6} & \multicolumn{2}{|c|}{7} & \multicolumn{2}{|c|}{8} & \multirow[b]{2}{*}{ Média } & \multirow[b]{2}{*}{ DP } \\
\hline & Média & DP & Média & DP & Média & DP & & \\
\hline Motricidade ampla & $110,57^{\mathrm{A}}$ & 8,42 & $98,49^{\mathrm{B}}$ & 7,78 & $89,46^{c}$ & 6,01 & 98,93 & 11,27 \\
\hline Equilíbrio & $117,66^{A}$ & 8,50 & $104,91^{\mathrm{B}}$ & 5,56 & $92,44^{c}$ & 4,51 & 104,23 & 11,91 \\
\hline Motricidade fina & $103,98^{A}$ & 13,42 & $97,24^{\mathrm{B}}$ & 9,44 & $87,64^{c}$ & 7,62 & 95,89 & 12,21 \\
\hline Geral & $110,50^{A}$ & 7,86 & $100,21^{\mathrm{B}}$ & 5,57 & $89,85^{c}$ & 4,28 & 99,64 & 10,23 \\
\hline
\end{tabular}

Comparação na linha: médias seguidas de letras distintas diferem significativamente por meio da Análise de Variância complementada pelo Teste de Comparações Múltiplas de Tukey, ao nível de significância de 5\%

Tabela 4. Classificação por idade. $(n=382)$

\begin{tabular}{|c|c|c|c|c|c|}
\hline \multirow[b]{2}{*}{ Quociente } & \multirow[b]{2}{*}{ Classificação } & \multicolumn{3}{|c|}{ Idade (anos) } & \multirow[t]{2}{*}{ Total } \\
\hline & & $\begin{array}{c}6 \\
(\%)\end{array}$ & $\begin{array}{c}7 \\
(\%)\end{array}$ & $\begin{array}{c}8 \\
(\%)\end{array}$ & \\
\hline \multirow{3}{*}{ Motricidade ampla } & Abaixo & 0 & 0,7 & 9,7 & 3,7 \\
\hline & Esperado & 89,5 & 99,3 & 90,3 & 93,2 \\
\hline & Acima & 10,5 & 0 & 0 & 3,1 \\
\hline \multirow{3}{*}{ Equilíbrio } & Abaixo & 0,9 & 0 & 3,7 & 1,6 \\
\hline & Esperado & 70,3 & 100 & 96,3 & 89,9 \\
\hline & Acima & 28,8 & 0 & 0 & 8,5 \\
\hline \multirow{3}{*}{ Motricidade fina } & Abaixo & 7,0 & 6,0 & 13,4 & 8,9 \\
\hline & Esperado & 89,5 & 94,0 & 86,6 & 90,1 \\
\hline & Acima & 3,5 & 0 & 0 & 1,0 \\
\hline
\end{tabular}

$\mathrm{QM}=$ Quociente Motor; Abaixo (QM <90); Esperado (QM=90 a 109); Acima (QM >109)

Para a evidência de validade convergente do TDI-HM, comparou-se o QM de cada construto com o QM do construto equivalente do EDM por meio da Correlação de Pearson. As análises indicaram uma correlação regular $(r=0,322 ; p<0,001)$ para a motricidade ampla e motricidade fina $(\mathrm{r}=$ $0,261(\mathrm{p}<0,001)$. 


\section{DISCUSSÃO}

O presente estudo levantou dados preliminares na busca de evidências de validade do instrumento TDI-HM em três dimensões consideradas fundamentais para o aprimoramento das HM, a saber: motricidade ampla, motricidade fina e equilíbrio $^{3,4,22,23}$. Há uma sequência do desenvolvimento de diversas habilidades motoras classificadas de acordo com a idade cronológica e a musculatura envolvida. Com o avanço da idade, a complexidade e as exigências envolvidas nas tarefas motoras também são incrementadas, a criança vai aprimorando as suas HM e melhorando seu desempenho ${ }^{1,3}$.

Baseado nisso o QM foi criado como resultado da avaliação estabelecida pelo TDI-HM, com cada tarefa composta por graus de dificuldade crescente e a eles atribuídos uma faixa etária correspondente. Essa é uma prática de correção comum a outros instrumentos internacionais já consagrados para avaliação de aspectos. É o caso, por exemplo, do Movement Assessment Battery For Children MABC- $I^{25}$, teste britânico usado para triagem, identificação e descrição de prejuízos no desempenho motor de crianças de 3 a 16 anos de idade; e da EDM16, que também apresenta padronização baseada em faixa etária: para cada idade é atribuída uma tarefa ${ }^{16,17}$.

Em relação aos resultados do domínio motor (resultado total), destaca-se a boa consistência interna apresentada, em especial, para a motricidade ampla com índice considerado muito bom. No entanto, houve redução no coeficiente quando analisadas as dimensões equilíbrio e motricidade fina separadamente. Isso pode ser justificado pelo número reduzido de itens do instrumento, pois o valor do coeficiente é fortemente influenciado pelo número de itens, sendo maior o valor do Alfa quanto maior o número de itens do teste ${ }^{18,19}$. De acordo com Pasquali ${ }^{19}$, instrumentos com mais de 14 itens devem apresentar valores superiores a 0,70 . A avaliação das HM no TDI-HM é composta por um total de 16 itens, e as dimensões isoladas são avaliadas por 6 itens de motricidade cada e 4 itens de equilíbrio. Mesmo assim, o coeficiente de equilíbrio e motricidade fina ficou acima de 0,67 , sendo esse um valor considerado aceitável quando envolve tão poucos itens $^{19}$.

Para os resultados de motricidade ampla, foi considerada a competência do movimento esperada para a faixa etária de acordo com a literatura ${ }^{3,4,23,24}$. Trata-se da fase dos movimentos fundamentais que são desenvolvidos entre 2 e 8 anos de idade, amadurecendo em eficiência e coordenação. Esses movimentos são divididos entre inicial, elementar e maduro ${ }^{3,23}$.

No TDI-HM, atribuiu-se para cada estágio uma idade correspondente, tendo, para isso, três tentativas. Os resultados mostraram que $96,3 \%$ das crianças que realizaram as duas tarefas (quicar e arremesso) estavam acima ou de acordo com o estágio esperado. Outros estudos apresentaram resultados semelhantes, como o de Matsunaga ${ }^{26}$ que traçou o perfil de escolares de 8 anos e encontrou 94\% como normal médio ou acima da média ${ }^{17,26}$. Esses altos índices justificam-se por ser o TDI-HM um instrumento para triagem, elaborado justamente com o intuito de verificar possíveis alterações do desenvolvimento. Espera-se que um grande percentual da amostra esteja de acordo com o esperado para sua idade, pois os sujeitos do estudo são classificados como crianças típicas, diferentemente de resultados encontrados em estudos realizados com amostras clínicas $^{6,9,23}$.

Em relação ao equilíbrio, a tarefa proposta foi composta de quatro itens, sendo esperado que as crianças de 6 a 8 anos consigam realizá-la de acordo com o grau de complexidade exigido, o que foi verificado em 98,4\% das crianças avaliadas. Mesmo as crianças mais novas tiveram um bom desempenho, pois apenas 0,9\% dos alunos de 6 anos não conseguiram atingir o nível esperado para a idade. $\mathrm{O}$ equilíbrio integra-se à motricidade ampla, no controle postural para execução do movimento, sendo responsável pela manutenção da cabeça e do tronco em posição vertical $^{1,2}$. Os resultados apresentados vão ao encontro dos achados de outros estudos, nos quais as crianças apresentaram desempenho semelhante na avaliação do equilíbrio $^{12,17}$. 
Tanto no que se refere à motricidade ampla quanto ao equilíbrio, estudos indicam que o fato de a criança explorar o ambiente por meio de atividades motoras, como exercício físico, jogos recreativos e atividades lúdicas, implica modificações no seu desenvolvimento físico e perceptivo-motor ${ }^{3,23,27}$. Essas atividades fazem parte do processo natural de desenvolvimento, capaz de permitir à criança desenvolver capacidades de adaptação e interação, as quais poderão, posteriormente, ser transferidas para diversas situações vivenciadas em seu cotidiano $0^{3,23,27}$.

Sobre os resultados referentes à motricidade fina, criou-se uma descrição normativa para o instrumento baseada no número de erros realizado pela criança ao executar cada uma das tarefas nos seus diferentes níveis de dificuldade. Considerou-se o ponto de corte fundamentado nos parâmetros da literatura já citados ${ }^{12,28}$. No presente estudo, 8,9\% dos alunos foram classificados como abaixo do esperado; esse número corrobora a prevalência de crianças dessa faixa etária com dificuldade de movimento ou risco de dificuldade, que, segundo a literatura, é menor que $25 \%{ }^{11,12,28}$. O percentual encontrado remete ao número de crianças que precisam ter um acompanhamento mais próximo e, talvez, uma avaliação mais aprofundada.

É possível que escolares que apresentam alterações motoras sejam subdiagnosticados em relação ao Transtorno do Desenvolvimento da Coordenação (TDC), acarretando prejuízo para início da trajetória acadêmica, na qual a demanda para a destreza, a velocidade de manipulação de objetos, a exatidão do movimento e a postura da mão são fundamentais para o desenvolvimento da escrita ${ }^{13,25}$. Justamente a motricidade fina é a dimensão do domínio motor mais estudado em crianças com idade escolar, pois as dificuldades no controle e na coordenação de movimentos podem ter impacto sobre a capacidade de aprendizagem e, como mencionado anteriormente, interferir no sucesso do desempenho escolar da criança ${ }^{13,19,29}$.

O percentual encontrado aponta para as estimativas de prevalência do TDC na população escolar. Mesmo sem um consenso, estudos internacionais apresentam prevalência de, aproximadamente, $5^{\circ}$ percentil para diagnóstico de TDC, entre $6^{\circ}$ e $15^{\circ}$ percentil para risco de TDC (r-TDC) e acima de $16^{\circ}$ para crianças típicas ${ }^{12,28,30}$.

No âmbito nacional, a prevalência está em torno de $8 \%$ para TDC e $19 \%$ para r-TDC ${ }^{28,31}$, ficando de acordo com o índice estimado (entre 6\% e 10\%) para todas as crianças em idade escolar ${ }^{10}$. A dificuldade em identificar possíveis atrasos é agravada, principalmente na rede pública, pela indisponibilidade de um instrumento de avaliação motora para crianças em fase de escolarização inicial, adaptado, validado e normatizado para população brasileira que seja livre de custo.

Ainda que outras análises estejam sendo realizadas para incrementar a busca de evidência de validade do TDI-HM, é importante considerar que as correlações estabelecidas entre o TDI-HM e o EDM foram inexpressivas, embora significativas. Os índices de correlação foram abaixo de 0,509, e apenas valores acima desse são aceitos para indicar que os instrumentos citados mensuram o mesmo construto ${ }^{32}$. No entanto, haja vista que a EDM não apresenta evidências de validade, apenas estudos de confiabilidade16, é questionável se a baixa magnitude das correlações seja decorrente de uma fragilidades da TDI-HM ou da EDM, eleita para este estudo como padrão concorrente por avaliar as mesmas dimensões da TDI-HM, por ser considerado também um teste de rastreio, por ser o instrumento elaborado e normatizado para população brasileira que contempla a faixa etária deste estudo e por ser bastante utilizado em pesquisas nacionais ${ }^{33,34}$.

Cite-se que vários estudos tiveram a dificuldade em correlacionar instrumentos de avaliação do DM por não serem adaptados para a população brasileira, comprometendo a discussão de seus resultados ${ }^{6,21,23}$. Uma alternativa seria pensar em utilizar outros testes como variáveis externas que avaliam construtos teóricos relacionados. Nesse caso, seriam esperadas correlações moderadas (entre 0,20 e 0,50) entre os testes ${ }^{32}$. 


\section{CONCLUSÕES}

Considerando todos os resultados apresentados, pode-se inferir que esse instrumento tem potencial para verificar se o desempenho das HM da criança está de acordo com os marcos do desenvolvimento esperados para a idade. Mesmo incipientes, os dados do presente estudo fomentam a discussão acerca do TDI-HM e demonstram que essa pode ser uma ferramenta viável para triagem do desenvolvimento, além de ter uma grande relevância ao oferecer um instrumento acessível e sem custo para ser utilizado em diferentes contextos, incluindo as redes públicas de saúde e de educação do Brasil.

\section{REFERÊNCIAS}

1. Bora LB, Cardoso VT, Toni PM. Assimetria Direita-Esquerda e Desenvolvimento Neuropsicomotor Humano. CES Psicolgía. 2019;12(1):54-68.

2. Hadders-Algra M. Early human motor development: From variation to the ability to vary and adapt. Neurosci Biobehav Rev. 2018;90(May):411-427.

3. Gallahue DL, Ozmun JC. Compreendendo o desenvolvimento motor - bebês, crianças, adolescentes e adultos. 7. ed. São Paulo: Editora P; 2013. 448 p.

4. Logan SW, Ross SM, Chee K, Stodden DF, Robinson LE. Fundamental motor skills: A systematic review of terminology. J Sports Sci. 2018;36(7):781-96

5. Guedes-Granzotti RB, Siqueira LS, Cesar CPHAR, Silva K, Domenis DR, Dornelas R, et al. Neuropsychomotor development and auditory skills in preschool children. J Hum Growth Dev. 2018;28(1):35-41.

6. Sandroni GA, Ciasca SM, Rodrigues SD. Avaliação da evolução do perfil motor de pré-escolares com necessidades educativas especiais após intervenção psicomotora breve. Rev Psicopedag. 2015;32(97):4-13.
7. Maurilia AP, Santos D. Aspectos biopsicossociais em escolares com atraso no desenvolvimento motor: um estudo longitudinal. J Hum Growth Dev. 2016;26(1).

8. Qi Y, Tan S, Sui M, Wang J. Supervised Physical Training Improves Fine Motor Skills of 5-Year-Old Children. Rev Bras Med do Esporte. 2018;24(1):9-12.

9. Arnoni JLB, Verdério BN, Pinto AMA, Rocha NACF. Efeito da intervenção com videogame ativo sobre o autoconceito, equilíbrio, desempenho motor e sucesso adaptativo de crianças com paralisia cerebral: estudo preliminar. Fisioter e Pesqui. 2018;25(3):294-302.

10. American Psychiatric Association. Manual diagnóstico e estatístico de transtornos mentais:DSM-5. 5. ed. Porto Alegre: Artmed Editora; 2014. 992 p.

11. Pulzi W, Rodrigues GM. Transtorno do Desenvolvimento da Coordenação: uma Revisão de Literatura. Rev Bras Educ Espec. 2015;21(3):433-44.

12. Nobre GC, Valentini NC, Ramalho MHS, Sartori RF. Self-efficacy profile in daily activities: Children at risk and with developmental coordination disorder. Pediatr Neonatol. 2019;60(6):662-668.

13. Madaschi V, Paula CS. Medidas de avaliação do desenvolvimento infantil: uma revisão da literatura nos últimos cinco anos. Cad. PPGDD. 2011;11(1):52-56.

14. Hansen J, Vieira V, Westphal JP, Vieira ML. Prontidão escolar: Uma experiência de pesquisa-intervenção. Arq Bras Psicol. 2012;64(2):115-29.

15. Favero E, Guerra D, Santos HLMM, Delazeri CM. O primeiro ano do ensino fundamental de nove anos: uma revisão teórica. Psicol Esc e Educ. 2017;1(3):397-406

16. Rosa Neto F. Manual de Avaliação Motora. Porto Alegre: Artmed; 2002. 144 p.

17. Rosa Neto F, Santos APM, Xavier RFC, Amaro KN. A Importância da avaliação motora em escolares: Análise da confiabilidade da Escala de Desenvolvimento 
Motor. Rev Bras Cineantropometria e 27. Adolph KE, Robinson SR. Motor Desempenho Hum. 2010;12(6):422-7. 18. American Educational Research Asso- developmental science: Cognitive procesciation. Standards for Educational and Psychological Testing. Washington, DC: AERA; 2014. 230 p.

19. Pasquali L. Psicometria: teoria dos testes na psicologia e na educação. Petrópolis, RJ: Vozes; 2017.

20. Coutinho MTC, Spessato BC, Valentini NC. Transtorno do Desenvolvimento a Coordenação: Prevalência e Dificuldades Motoras de Escolares da Cidade de Porto Alegre. An do XVII Congr Bras Ciências do Esporte e IV Congr Int Ciências do Esporte Porto Alegre. 2011;17:1-9.

21. Valentini NC, Ramalho MH, Oliveira MA. Movement Assessment Battery for Children-2: Translation, reliability, and validity for Brazilian children. Res Dev Disabil. 2014;35(3):733-740.

22. Beltrame TS, Capistrano R, Alexandre JM, Lisboa T, Andrade RD, Felden ÉPG Prevalência do Transtorno do Desenvolvimento da Coordenação em uma Amostra de Crianças Brasileiras. Cad Ter Ocup da UFSCar. 2017;25(1):105-113.

23. Sá MV, Lara S, Graup S, Balk RDS Sasso RR. Análise do desenvolvimento motor e da atenção de crianças submetidas a um programa de intervenção psicomotora. ConScientiae Saúde. 2018;17(2):187-195.

24. Flôres FS, Rodrigues LP, Copetti F, Lopes F, Cordovil R. Affordances for Motor Skill Development in Home, School, and Sport Environments: A

Narrative Review. Percept Mot Skills. 34. Souza A, Souza WC, Reiser FC, Ruse2019;126(3):366-388

25. Henderson SE, Sugden DA. Movement renhas LPG. Perfil do Desenvolvimento Movement Motor de Alunos de Oito Anos de Escola Assessment Battery for Children. 2nd ed. Públicas Estaduais de São Bento do Sul London: Psychological Corporation; 2007. - SC. Rev Pesqui em Fisioter. 2015;5(3).

26. Matsunaga NY, Pereira K, Marcacine PR, Castro SS, Walsh IAP. Efeitos de atividades psicomotoras no desenvolvimento motor de pré-escolares de cinco anos de idade. ConScientiae Saúde. 2016;15(1):3843. Inc; 2015. p. 137-157.

30. Cabello LB. Diagnostic challenge and development. In: Lamb, ME, Lerner, RM (Eds.). Handbook of child psychology and

$\frac{11}{\text { Machado et al }}$ ses. Hoboken, NJ, US: John Wiley \& Sons

28. Valentini NC, Coutinho MTC, Pansera SM, Santos VAP, Vieira JLL, Ramalho $\mathrm{MH}$, et al. Prevalência de déficits motores e desordem coordenativa desenvolvimental em crianças da região Sul do Brasil. Rev Paul Pediatr. 2012;30(3):377-384.

29. Espíndola JA. Avaliação das tarefas de coordenação óculo-manuais de motricidade fina de baterias motoras e desempenho escolar. Universidade do Estado de Santa Catarina - Udesc; 2015. importance of the clinical approach of the Developmental Coordination Disorder. Arch Argent Pediatr. 2019;117(3).

31. Lima SS, Cavalcante LIC, Costa EF. Triagem do desenvolvimento neuropsicomotor de crianças brasileiras: uma revisão sistemática da literatura. Fisioter e Pesqui. 2016;23(3):336-342

32. Nunes CHSS, Primi R. Aspectos técnicos e conceituais da ficha de avaliação dos testes psicológicos. In: Conselho Federal de Psicologia, editor. Conselho Federal de Psicologia, Avaliação psicológica: diretrizes na regulamentação da profissão. Brasília: CFP; 2010. p. 101-128.

33. Costa AGS, Cavalcante JL. Desenvolvimento da motricidade fina em crianças com desnutrição crônica. Cad Bras Ter Ocup. 2019;27(1):54-60. nhack MC, Moncada-Jiménez J, Mascarenhas LPG. Perfil do Desenvolvimento 